\title{
Radiation Recall Dermatitis Triggered by Prednisone
}

\author{
Jessica Galant-Swafford, MD; Meng Chen, MD; Sandra C. Christiansen, MD
}

\section{PRACTICE POINTS}

- Consider the diagnosis of radiation recall dermatitis for a skin eruption that occurs in the same location as prior radiation exposure.

- Prednisone may be a trigger for radiation recall dermatitis in patients with sensitization to cross-reactive topical steroids such as tixocortol pivalate.

- Radiation therapy may prime the skin for a future inflammatory response by upregulating proinflammatory cytokines that persist after the conclusion of treatment.

To the Editor:

A 69-year-old woman presented to the allergy clinic for evaluation of a rash on the left breast. The patient had a history of breast cancer that was treated with a lumpectomy followed by external beam radiation therapy (total dose, 6000 cGy) to the lateral aspect of the left breast approximately 4 years prior. She developed acute breast dermatitis from the radiation, which was self-treated with over-the-counter hydrocortisone cream. The patient subsequently developed a blistering skin eruption over the area where she applied the cream. She did not recall the subtype of hydrocortisone she used (butyrate and acetate are available over-the-counter). She discontinued the hydrocortisone and was started on triamcinolone cream $0.1 \%$, which was well tolerated, and the rash resolved.

The patient had a history of a similar reaction to hydrocortisone butyrate after blepharoplasty approximately 10 years prior to the current presentation, characterized by facial erythema, pruritus, and blistering. A patch test confirmed reactivity to hydrocortisone-17-butyrate and tixocortol pivalate. However, a skin-prick test for hydrocortisone acetate cream $1 \%$ was negative.

Subsequently, the patient developed acute-onset dyspepsia, gnawing epigastric pain, regurgitation, and bloating. A diagnosis of eosinophilic gastritis was established via biopsy, which found increased eosinophils in the lamina propria (>50 eosinophils per high-power field). Helicobacter pylori was not identified. She was started on the proton-pump inhibitor dexlansoprazole but symptoms did not improve. Her other medications included benazepril, alprazolam as needed, vitamin $\mathrm{D}$, and magnesium. The patient subsequently was started on a trial of oral prednisone $40 \mathrm{mg} / \mathrm{d}$. Three days after initiation, she developed an erythematous macular rash over the left breast.

The next day she presented to the allergy clinic. Physical examination of the left breast revealed a $20 \times 10-\mathrm{cm}$, nipple-sparing patch of well-demarcated erythema without fluctuance or overlying lesions. The area of erythema overlapped with the prior radiation field based on radiation marker tattoos and the lumpectomy scar (Figure). There was no evidence to suggest inflammation of deeper tissue or the pectoral muscles. Vital signs were normal, and the remainder of the examination was unremarkable, including breast, lymph node, and complete skin examinations.

At evaluation, the differential diagnosis included contact dermatitis, fixed drug eruption, infection, tumor recurrence with overlying skin changes, and radiation recall dermatitis. Given that the dermatitis had developed

Drs. Galant-Swafford and Christiansen are from the Department of Internal Medicine, Division of Rheumatology, Allergy \& Immunology, University of California, San Diego. Dr. Chen is from the Department of Medicine, Division of Pulmonary, Allergy \& Critical Care Medicine, Stanford University, California.

The authors report no conflict of interest.

This work was supported by National Institutes of Health grant T32 Al 007469.

Correspondence: Jessica Galant-Swafford, MD, 8899 University Center Ln, Ste 230, San Diego, CA 92122 (jgalantswafford@health.ucsd.edu). 


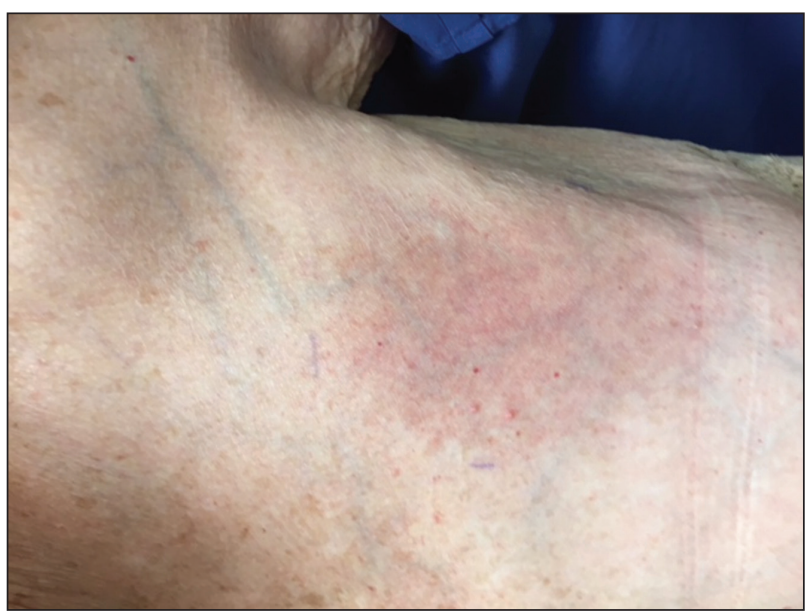

Radiation recall dermatitis presenting as a pruritic erythematous rash on the upper left breast in an area that overlapped with a prior radiation field.

at the site of previously irradiated skin in the absence of fever or an associated mass, the presentation was thought to be most consistent with radiation recall dermatitis.

Oral prednisone was discontinued, and the dermatitis spontaneously improved in a few weeks. Given the patient's test results and prior tolerance to triamcinolone, eosinophilic gastroenteritis was treated with triamcinolone acetonide $40 \mathrm{mg}$ via intramuscular injection, which was well tolerated.

Radiation recall dermatitis is an acute inflammatory reaction over an area of skin that was previously irradiated. It is most often triggered by chemotherapy agents and occurs in as many as 9\% of patients who receive chemotherapy after radiation. ${ }^{1}$ Commonly implicated chemotherapy agents include anthracyclines, taxanes, antimetabolites, and alkylating agents. Newer targeted cancer treatments also have been reported to trigger radiation recall dermatitis, including epidermal growth factor receptor inhibitors, vascular endothelial growth factor receptor inhibitors, mammalian target of rapamycin inhibitors, and anti-programmed cell death protein 1 monoclonal antibodies. $^{2-5}$ Radiation recall dermatitis also has been reported to be triggered by intravenous contrast dye. ${ }^{6}$

The clinical presentation of radiation recall dermatitis ranges from mild rash to skin necrosis and desquamation. Patients often report pruritus or pain in the affected area. The US National Cancer Institute's Common Terminology Criteria for Adverse Events (CTCAE) includes a 5-point scale for grading the severity of radiation recall dermatitis: grade 1, faint erythema or dry desquamation; grade 2, moderate to brisk erythema or patchy moist desquamation, mostly confined to skin folds and creases; grade 3, moist desquamation in areas other than skin folds and creases, with bleeding induced by minor trauma or abrasion; grade 4, skin necrosis or ulceration of full-thickness dermis, with spontaneous bleeding; grade 5, death. ${ }^{7}$
Based on these criteria, our patient had grade 2 radiation recall dermatitis.

In addition to cutaneous inflammation, additional sites can be inflamed, including the gastrointestinal tract, lungs, and oral mucosa. Cases of myocarditis, sialadenitis, and cystitis also have been reported. ${ }^{7}$

Radiation recall dermatitis can occur even if dermatitis did not occur upon initial treatment. The inflammatory reaction can occur weeks or years after initial irradiation. A study evaluating targeted chemotherapy agents found the median time from initiation of chemotherapy to radiation recall dermatitis was 16.9 weeks (range, 1-86.9 weeks). Inflammation usually lasts approximately 1 to 2 weeks but has been reported to persist as long as 14 weeks. ${ }^{8}$ Withdrawal of the offending agent in addition to administration of corticosteroids or nonsteroidal anti-inflammatory agents typically results in clinical improvement. Histology on skin biopsy is nonspecific and can reveal mixed infiltrates. ${ }^{7}$

The pathophysiology of radiation recall dermatitis remains unknown; the condition might be an idiosyncratic drug reaction. It has been hypothesized that prior radiation lowers the threshold for an inflammatory reaction, an example of Ruocco immunocompromised cutaneous districts, in which a prior injury at a cutaneous site increases the likelihood of opportunistic infection, tumor, and immune reactions. ${ }^{9}$ Because radiation can induce expression of inflammatory cytokines, such as IL-1, IL-6, platelet-derived growth factor $\beta$, and tumor necrosis factor $\alpha$, cells in irradiated areas can continue to secrete low levels of these cytokines after radiation therapy, thus priming an inflammatory reaction in the future. ${ }^{10}$ An alternative theory is that radiation induces mutations within surviving stem cells, rendering them unable to tolerate or unusually sensitive to subsequent chemotherapy and cytotoxic drugs. However, this premise would not explain how noncytotoxic drugs also can trigger radiation recall dermatitis, as described in our case. ${ }^{11}$

Prednisone-triggered radiation recall dermatitis is curious, as corticosteroids are used to treat the condition. Corticosteroids are classified by their chemical structure, and patch testing can be used to distinguish allergies across the various classes. Hydrocortisone acetate, tixocortol pivalate, and prednisone are class A steroids; they have no substitutions in the D ring but have C21 shortchain esters. Hydrocortisone-17-butyrate is a class D2 steroid; it does not have any C16 methyl substitutions or halogenations, possesses a C17 long-chain ester, and can be with or without a C21 side chain. Class A steroids are cross-reactive with each other and also are known to crossreact with D2 steroids. In our patient, therefore, the patch test-confirmed allergy to tixocortol pivalate could explain the hypersensitivity to prednisone via cross-reactivity. ${ }^{12}$

In contrast, triamcinolone is a class B steroid, which has a C16,17-cis-diol or -ketal. Other than budesonide, which can cross-react with D2 steroids, class B steroids do not cross-react with hydrocortisone or prednisone. 
Triamcinolone does not usually cross-react with D2 corticosteroids, which likely explains why our patient was later able to tolerate triamcinolone to treat eosinophilic gastrointestinal tract disease.

In summary, we present a case of radiation recall dermatitis triggered by prednisone. Radiation can prime an area for a future inflammatory response by upregulating proinflammatory cytokines or triggering stem cell mutation. In our case, clinical reactivity to hydrocortisone17-butyrate and sensitization to tixocortol pivalate via patch testing could have increased the likelihood of a reaction with prednisone use due to cross-reactivity. This case instructs dermatologists, allergists, and oncologists to be aware of prednisone as a potential trigger of radiation recall dermatitis.

\section{REFERENCES}

1. Kodym E, Kalinska R, Ehringfeld C, et al. Frequency of radiation recall dermatitis in adult cancer patients. Onkologie. 2005;28:18-21.

2. Seidel C, Janssen S, Karstens JH, et al. Recall pneumonitis during systemic treatment with sunitinib. Ann Oncol. 2010;21:2119-2120.

3. Togashi $\mathrm{Y}$, Masago K, Mishima M, et al. A case of radiation recall pneumonitis induced by erlotinib, which can be related to high plasma concentration. J Thorac Oncol. 2010;5:924-925.
4. Bourgier C, Massard C, Moldovan C, et al. Total recall of radiotherapy with mTOR inhibitors: a novel and potentially frequent side-effect? Ann Oncol. 2011;22:485-486.

5. Korman AM, Tyler KH, Kaffenberger BH. Radiation recall dermatitis associated with nivolumab for metastatic malignant melanoma. Int J Dermatol. 2017;56:e75-e77.

6. Lau SKM, Rahimi A. Radiation recall precipitated by iodinated nonionic contrast. Pract Radiat Oncol. 2015;5:263-266.

7. US Department of Health and Human Services. Common Terminology Criteria for Adverse Events (CTCAE) Version 5.0. https://ctep.cancer.gov/protocoldevelopment/electronic_applications /docs/CTCAE_v5_Quick_Reference_8.5x11.pdf. Published November 27, 2017. Accessed June 10, 2020.

8. Levy A, Hollebecque A, Bourgier C, et al. Targeted therapy-induced radiation recall. Eur J Cancer. 2013;49:1662-1668.

9. PiccoloV, Baroni A, Russo T, et al. Ruocco's immunocompromised cutaneous district. Int J Dermatol. 2016;55:135-141.

10. Johnson CJ, Piedboeuf $\mathrm{P}$, Rubin $\mathrm{P}$, et al. Early and persistent alterations in the expression of interleukin-1 alpha, interleukin-1 beta and tumour necrosis factor alpha mRNA levels in fibrosis-resistant and sensitive mice after thoracic irradiation. Radiat Res. 1996;145: 762-767.

11. Azira D, Magné N, Zouhair A, et al. Radiation recall: a well recognized but neglected phenomenon. Cancer Treat Rev. 2005;31:555-570.

12. Jacob SE, Steele T. Corticosteroid classes: a quick reference guide including patch test substances and cross-reactivity. J Am Acad Dermatol. 2006;54:723-727. 